ORIGINAL ARTICLE

\title{
Increased inflammation and altered macrophage chemotactic responses caused by two ultrafine particle types
}

\author{
L C Renwick, D Brown, A Clouter, K Donaldson
}

Occup Environ Med 2004;61:442-447. doi: 10.1136/oem.2003.008227

\begin{abstract}
See end of article for authors' affiliations

.....................

Correspondence to: Professor K Donaldson, ELEGI Colt Laboratory, Wilkie Building, University of Edinburgh, Medical School, Teviot Place, Edinburgh EH9 8AG, UK; ken.donaldson@ed.ac.uk
\end{abstract}

Accepted 16 May 2003

\begin{abstract}
Background: Ultrafine particles have been hypothesised to be an important contributing factor in the toxicity and adverse health effects of particulate air pollution $\left(\mathrm{PM}_{10}\right)$ and nanoparticles are used increasingly in industrial processes.

Aims: To compare the ability of ultrafine and fine particles of titanium dioxide and carbon black to induce inflammation, cause epithelial injury, and affect the alveolar macrophage clearance functions of phagocytosis and chemotaxis in vivo.

Methods: Rats were instilled with fine and ultrafine carbon black and titanium dioxide. Inflammation was quantified by bronchoalveolar lavage; the ability of the macrophages to phagoytose indictor fluorescent beads and to migrate towards aC5a were determined.

Results: Ultrafine particles induced more PMN recruitment, epithelial damage, and cytotoxicity than their fine counterparts, exposed at equal mass. Both ultrafine and fine particles significantly impaired the phagocytic ability of alveolar macrophages. Only ultrafine particle treatment significantly enhanced the sensitivity of alveolar macrophages to chemotact towards C5a.

Conclusions: Ultrafine particles of two very different materials induced inflammation and epithelial damage to a greater extent than their fine counterparts. In general, the effect of ultrafine carbon black was greater than ultrafine titanium dioxide, suggesting that there are differences in the likely harmfulness of different types of ultrafine particle. Epithelial injury and toxicity were associated with the development of inflammation after exposure to ultrafines. Increased sensitivity to a C5a chemotactic gradient could make the ultrafine exposed macrophages more likely to be retained in the lungs, so allowing dose to accumulate.
\end{abstract}

U trafine particles, or nanoparticles, describe a class of particles that have only relatively recently been recognised as a potential hazard to the lungs. ${ }^{1}$ These are particles below about $100 \mathrm{~nm}$ that are produced by fuel combustion ${ }^{2}{ }^{3}$ as well as by other industrial processes. ${ }^{4}$ The exceptional toxicity of ultrafine particles compared to fine particles of the same material has now been shown for a number of diverse materials..$^{5-7}$ This increased ultrafine particle mediated toxicity has been related to their small size, their corresponding large surface areas per unit mass, and surface associated free radical generating systems. $^{7-9}$

The potential risks from nanoparticles come from two main sources: (1) a risk to the general population from ultrafine particles derived largely from combustion of fuel ${ }^{2}{ }^{3}$ which are a component of $\mathrm{PM}_{10}$ air pollution; ${ }^{10}$ and (2) a risk to selected worker populations involved in producing nanoparticles for various industrial uses. ${ }^{4}$ The new recognition of the potential hazard from ultrafine particles has stimulated some research into the mechanism of their effects, ${ }^{1}$ but this is still in its infancy and much remains to be understood regarding the mechanism of lung injury and inflammation caused by very small particles.

We have shown previously in vitro that ultrafine particles impaired the ability of macrophages to subsequently phagocytose latex indicator particles to a greater extent than fine particles of the same material. ${ }^{11}$ In the present study, we utilised a rat instillation model to compare the ability of ultrafine and fine particles of the same material to induce inflammation, cause epithelial damage, and affect the macrophage clearance functions of phagocytosis and chemotaxis.

\section{MATERIALS AND METHODS}

\section{Chemicals and reagents}

Chemicals and reagents were purchased from Sigma Chemicals Company (Poole, Dorset, UK) unless otherwise stated.

\section{Animals}

Male Wistar rats were supplied by Charles River, UK and weighed between $370 \mathrm{~g}$ and $470 \mathrm{~g}$ at the time of use.

\section{Particles}

The particles used in this study were: (a) fine carbon black (CB), obtained from H. Haeffner \& Co. Ltd (Chepstow, UK) as Huber 990; (b) ultrafine or nanoparticles carbon black (UCB), obtained from Degussa as Printex 90; (c) fine titanium dioxide $\left(\mathrm{TiO}_{2}\right)$, provided by Tioxide Ltd; and (d) ultrafine titanium dioxide $\left(\mathrm{UTiO}_{2}\right)$, obtained from Degussa. Table 1 shows the mean diameter and surface area of particulates. Surface area was determined by Brunauer, Emmett, and Teller (BET) analysis, and diameter was measured using transmission electron microscopy.

\section{Particulate instillation}

Particles were suspended in sterile saline (Baxter, UK) at the concentration required, and sonicated (Grant Ultrasonic Bath XB6) for 20 minutes prior to use. Rats were lightly

Abbreviations: $B A L$, bronchoalveolar lavage; $C B$, carbon black; GGT, $\gamma$-glutamyl transpeptidase; LDH, lactate dehydrogenase; $P M$, particulate matter; PMN, polymorphonuclear neutrophils; UCB, ultrafine carbon black; UTiO 2 , ultrafine titanium dioxide; ZAS, zymosan activated serum 
Table 1 Mean diameters and surface area of ultrafine and fine particles

\begin{tabular}{llc}
\hline Particle type & Mean diameter $(\mathbf{n m})$ & Surface area $\left(\mathbf{m}^{2} / \mathbf{g}\right)$ \\
\hline $\mathrm{CB}$ & 260.2 & 7.9 \\
$\mathrm{UCB}$ & 14.3 & 253.9 \\
$\mathrm{TiO}_{2}$ & 250.0 & 6.6 \\
$\mathrm{UTiO}_{2}$ & 29.0 & 49.78 \\
\hline
\end{tabular}

Surface area was determined by nitrogen adsorption, whereas size was Surface area was determined by nitrogen adsorption
measured using transmission electron microscopy.

anaesthetised with halothane and instilled intratracheally with $125 \mu \mathrm{g}$ or $500 \mu \mathrm{g}$ of particles suspended in $0.5 \mathrm{ml}$ of sterile saline. Control animals were instilled with $0.5 \mathrm{ml}$ of saline only. Rats were sacrificed 24 hours post-exposure.

\section{Bronchoalveolar lavage}

Rats were killed by a pentobarbitone overdose and their body weight recorded. The chest cavity was opened and the trachea cannulated using a luer port cannula (Scientific Laboratory Supplies Ltd, Nottingham, UK). Lungs were removed and lavaged with $4 \times 8 \mathrm{ml}$ of sterile saline (Baxter). The first lavage was retained in a separate tube for cell free analysis of lactate dehydrogenase $(\mathrm{LDH})$, protein, and $\gamma$ glutamyl transpeptidase estimations. Bronchoalveolar lavage (BAL) leucocytes were isolated by centrifugation (900 g for 10 minutes). The lavage fluid was discarded, except for the first lavage that was designated BAL fluid, aliquoted, and frozen at $-80^{\circ} \mathrm{C}$, until required. Cell pellets were resuspended in $1 \mathrm{ml}$ of culture medium (RPMI 1640 medium supplemented with $1 \%$ penicillin/streptomycin, 1\% L-glutamine, 5\% Ultroser G, and 0.5\% amphotericin B; Gibco BRL, Paisley, UK) and kept on ice. Cell counts and viability were determined using a haemocytometer and trypan blue dye exclusion. Cells were then resuspended in culture medium according to the concentration required. Cell cultures were kept in a humidified incubator at $37^{\circ} \mathrm{C}$ with $95 \%$ air and $5 \%$ $\mathrm{CO}_{2}$, unless otherwise stated.

\section{BAL cell counting}

The degree of inflammation induced by the instilled particles was estimated by the total numbers of neutrophils recruited into the rat lung. Cytocentrifuge smears were prepared, air dried, stained with Diffquick (Raymond A Lamb, London, UK) and differential counts of BAL leucocytes was carried out. Five hundred cells per slide were counted and the results expressed as a percentage of the total number of BAL leucocytes.

\section{Phagocytosis}

The phagocytic ability of the isolated primary rat macrophages was assessed by measuring their ability to phagocytose $2 \mu \mathrm{m}$ fluorescent latex beads. ${ }^{11 a}$ Cell suspensions $(200 \mu \mathrm{l}$ of $5 \times 10^{5}$ cells $/ \mathrm{ml}$ ) were dispensed into wells (triplicate) of a 96 well plate and incubated for four hours. The medium was removed and replaced with culture medium $(200 \mu \mathrm{l})$ containing $2 \mu \mathrm{m}$ latex beads at a bead to macrophage ratio of $5: 1$. Cells were incubated with the beads for approximately 18 hours. Beads not phagocytosed were removed by discarding the culture medium and washing the cells with PBS $(\times 2)$. The cells were air dried and stained with Diffquick in order to distinguish the alveolar macrophages from the other BAL leucocytes. Macrophages which phagocytosed two or more of the latex beads were counted as being phagocytic. Five hundred cells per well were counted, and the results expressed as a percentage of the total number of macrophages counted.

\section{Chemotaxis assays}

Zymosan A was suspended in saline $(10 \mathrm{mg} / \mathrm{ml})$ and sonicated for five minutes to disperse the particles, prior to use. Serum was obtained from male Wistar rats by centrifuging clotted blood at $2500 \mathrm{rpm}$ for 10 minutes. Zymosan suspension was added to the serum at a concentration of $1 \mathrm{mg} / \mathrm{ml}$ and incubated in a shaking water bath for one hour at $37^{\circ} \mathrm{C}$ to obtain $\mathrm{C} 5 \mathrm{a}$-rich serum. For control the serum was incubated with saline only. The zymosan activated serum (ZAS) and control serum were inactivated at $56^{\circ} \mathrm{C}$ for 30 minutes. Zymosan particles were then removed from the serum by centrifugation. Serum was aliquoted and frozen at $-80^{\circ} \mathrm{C}$ until required. Both the ZAS and control serum were diluted to $10 \%$ with culture medium for the chemotaxis assays.

The potential of isolated alveolar macrophages to migrate towards the standard chemotaxin C5a (zymosan activated serum) was determined using a 96 well chemotaxis chamber (Model AB96; Neuro Probe Inc., Gaithersburg, MD). The upper and lower chambers were separated by a $5 \mu \mathrm{m}$ pore polycarbonate filter. Briefly, $30 \mu \mathrm{l}$ of ZAS was dispensed into wells (triplicate) of the lower compartment and $200 \mu \mathrm{l}$ of cell suspension $\left(6 \times 10^{5}\right.$ cells $)$ were transferred to wells (triplicate) of the upper compartment. The chamber was incubated in a humidified incubator for 3.5 hours. The filter was removed, air dried, stained with Diffquick, and air dried again. The adherent cells were displaced from the filter by washing the cells with water and then gently scraping them from the top side of the filter. The filter was air dried and the absorbance read at $550 \mathrm{~nm}$ using an MRX Microplate reader. For a control, macrophages from saline instilled animals were also assessed in their ability to migrate towards both the control serum (serum/saline suspension) and culture medium. There was no significant difference between the chemotactic potential of the two controls (data not shown). Thus, the culture medium was used as a standard measurement of random macrophage migration. The absorbance value of the stained filter was subtracted from both the test and control samples. The chemotactic potential of the isolated macrophages was then calculated as: test sample - control (culture medium). Test results were expressed as an absorbance at $550 \mathrm{~nm}$.

\section{Epithelial damage}

Lung epithelial damage was estimated by $\gamma$-glutamyl transpeptidase (GGT) activity in BAL fluid, using a modified version of the Sigma diagnostic kit (no. 545). ${ }^{12}$ The kit was adapted for use on a 96 well plate. BAL fluid $(25 \mu \mathrm{l})$ was transferred into triplicate wells of a 96 well plate. GGTP substrate solution $(25 \mu \mathrm{l})$ was added to each well and to three empty wells, as a blank. The plate was incubated for 20 minutes at $37^{\circ} \mathrm{C}$. The reaction was suspended by the addition of $100 \mu \mathrm{l}$ of acetic acid. In a timed sequence, sodium nitrite solution $(50 \mu \mathrm{l})$ was added to all wells followed by ammonium sulphamate $(50 \mu \mathrm{l})$ and finally, napthylethylenediamine solution $(50 \mu \mathrm{l})$. The resulting colour was read using an MRX Microplate reader at $540 \mathrm{~nm}$. A standard curve (0-100 units/ml) was set up using the Sigma supplied calibration solution. GGT activity was determined from the standard curve.

\section{Total protein}

The permeability of the lung epithelium was determined by the concentration of protein in the BAL fluid. This was carried out using a modified version of the Sigma bicinchoninic acid protein assay kit. ${ }^{13}$ The kit was adapted for use on a 96 well plate. Briefly, $10 \mu \mathrm{l}$ of BAL fluid was transferred into triplicate wells of a 96 well plate. To each well, $190 \mu \mathrm{l}$ of reagent $(10 \mathrm{ml}$ of bicinchoninic acid and $0.2 \mathrm{ml}$ copper II 
sulphate) was added and the plate incubated for 30 minutes at $37^{\circ} \mathrm{C}$. The absorbance was read at $540 \mathrm{~nm}$ using an MRX Microplate reader. The concentration of protein was determined by comparison with a standard curve for bovine serum albumin $(0-1000 \mu \mathrm{g} / \mathrm{ml})$. The blank consisted of the reagent only and was treated as the test samples.

\section{Cytotoxicity}

The degree of cytotoxicity induced by the instilled particles was determined by LDH activity in the BAL fluid. This was carried out using a modified method of the Sigma LDH diagnostic kit (procedure no. 500). The kit was adapted for use on a 96 well plate. BAL fluid $(10 \mu \mathrm{l})$ was aliquoted into wells (triplicate) of a 96 well plate. To each well, $50 \mu \mathrm{l}$ of $0.75 \mathrm{mM}$ sodium pyruvate solution containing NADH ( $1 \mathrm{mg} /$ $\mathrm{ml}$ ) was added, and the plate incubated for 30 minutes at $37^{\circ} \mathrm{C}$. 2,4-Dinitrophenylhydrazine was dissolved in $1 \mathrm{M} \mathrm{HCl}$ at a concentration of $20 \mathrm{mg} / \mathrm{dl}$, and warmed to room temperature. The reagent $(50 \mu \mathrm{l})$ was then added to each well and the plate incubated for 20 minutes at room temperature. Finally, $50 \mu \mathrm{l}$ of $4 \mathrm{M} \mathrm{NaOH}$ was added and the plate incubated for a further five minutes at room temperature. The resulting colour was read using an MRX Microplate reader. A standard curve (0-2000 units/ml) was prepared using the Sigma supplied calibration solution. LDH activity was determined from the standard curve.

\section{Statistical analysis}

Results were calculated as the mean value (SEM) of three separate experiments. The significance of the results was statistically analysed by ANOVA one way analysis of variance with Tukey's multiple comparison for pairwise comparisons.

\section{RESULTS}

\section{Inflammation}

Table 2 shows the total number of cells in the lavage following the different treatments. There were only modest increases in the total cell number and there were no significant differences from the control, therefore the proportion (\%) of PMN adequately describes the PMN influx into the BAL (fig 1). Instillation of $125 \mu \mathrm{g}$ of UCB induced significantly greater $(\mathrm{p}<0.05)$ neutrophil recruitment compared to either the saline control or the other test particles (fig 1). Significant increases in neutrophil numbers $(\mathrm{p}<0.001)$ also occurred following exposure to the highest dose of $\mathrm{UTiO}_{2}$ and UCB $(500 \mu \mathrm{g})$. In contrast, instillation of the same mass of fine $\mathrm{TiO}_{2}$ and CB produced no significant change in the proportion of neutrophils compared to the saline control.

\section{Epithelial damage}

Injury to both epithelial type II and Clara cells was assessed as GGT activity in BAL fluid (fig 2). Instillation of $500 \mu \mathrm{g}$ of $\mathrm{UTiO}_{2}$ and both high and low doses of UCB induced significant $(p<0.001)$ epithelial damage as shown by the increase in GGT activity, above the saline control. This effect

Table 2 Total number of BAL leucocytes 24 hours after instillation of test particles (millions)

\begin{tabular}{lll}
\hline & $125 \mu \mathrm{g}$ & $500 \mu \mathrm{g}$ \\
\hline $\mathrm{TiO}_{2}$ & $7.03(0.67)$ & $7.57(0.27)$ \\
$\mathrm{UTiO}_{2}$ & $6.77(0.21)$ & $6.78(0.76)$ \\
$\mathrm{CB}$ & $6.23(1.21)$ & $8.01(0.91)$ \\
$\mathrm{UCB}$ & $6.83(1.45)$ & $8.61(1.17)$ \\
Saline & $6.29(1.05)$ & \\
\hline
\end{tabular}

Results are the mean (SEM) of three separate experiments. Asterisks denote significant differences from the saline control $\left({ }^{*} p<0.01\right)$.

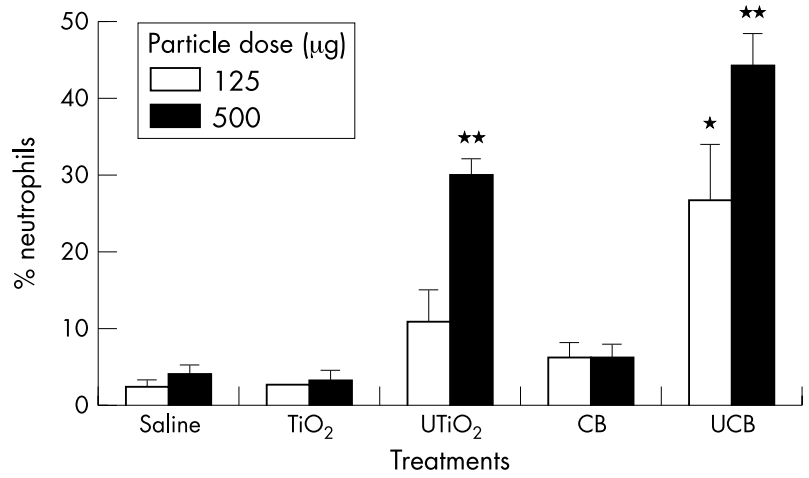

Figure 1 Neutrophil influx into the rat lung, 24 hours after instillation of test particles $(125 \mu \mathrm{g}$ or $500 \mu \mathrm{g})$. Results are the mean (SEM) of three separate experiments. Asterisks indicate significant differences from the saline control ( $\left.{ }^{*} \mathrm{p}<0.05 ;{ }^{* *} \mathrm{p}<0.001\right)$.

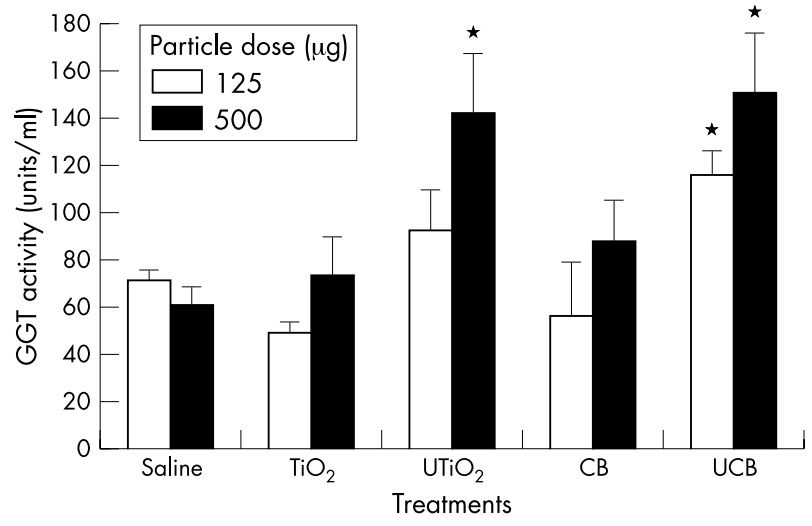

Figure 2 GGT activity in BAL fluid 24 hours after instillation of test particles $(125 \mu \mathrm{g}$ or $500 \mu \mathrm{g})$. Results are the mean (SEM) of three separate experiments. Asterisks indicate significant differences from the saline control ( $\left.{ }^{*} \mathrm{p}<0.05\right)$.

was significantly greater $(\mathrm{p}<0.05)$ than that of the fine particles, which had no effect at either dose.

\section{Total protein}

Changes in lung permeability were determined as total protein in the BAL fluid (fig 3). No low dose particle treatment had any effect on the permeability of the lung epithelium. Increased lung permeability was observed

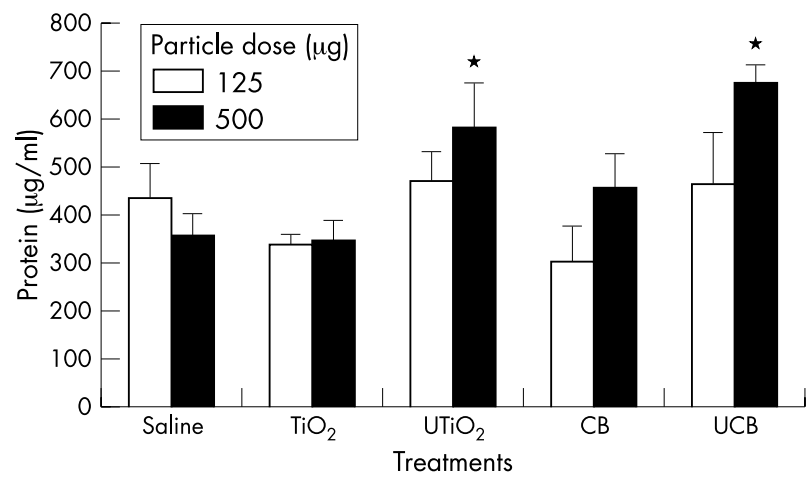

Figure 3 Concentration of protein in BAL fluid 24 hours after instillation of test particles $(125 \mu \mathrm{g}$ or $500 \mu \mathrm{g})$. Results are the mean (SEM) of three separate experiments. Asterisks indicate significant differences from the saline control (* $\mathrm{p}<0.001)$. 


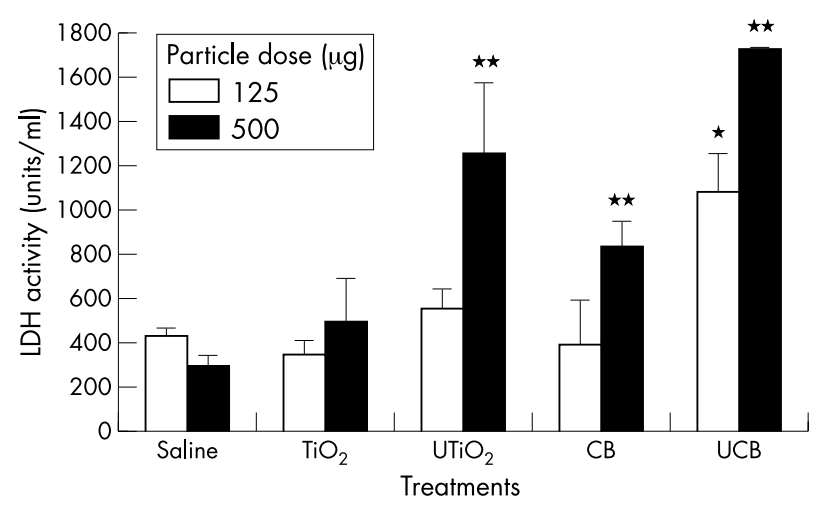

Figure 4 LDH activity in BAL fluid 24 hours after instillation of test particles $(125 \mu \mathrm{g}$ or $500 \mu \mathrm{g})$. Results are the mean (SEM) of three separate experiments. Asterisks indicate significant differences from the saline control ( $\left.\mathrm{p}<0.05 ;{ }^{* *} \mathrm{p}<0.001\right)$.

following exposure to the highest dose of $\mathrm{UTiO}_{2}$ and UCB $(p<0.001)$, but the same mass of their fine counterparts had no effect on the protein level.

\section{Cytotoxicity}

Cell death was estimated by LDH activity in BAL fluid (fig 4). Significantly increased LDH $(\mathrm{p}<0.05)$ was found following exposure to $125 \mu \mathrm{g}$ of UCB. At the higher dose all particles except $\mathrm{TiO}_{2}$ were significantly cytotoxic $(\mathrm{p}<0.001)$, but this toxic effect was greatest following exposure to the ultrafine particles $\left(\mathrm{UCB}\right.$ and $\left.\mathrm{UTiO}_{2}\right)(\mathrm{p}<0.001)$. Although the CB was significantly cytotoxic at $500 \mu \mathrm{g}$, the mean LDH was only about half the mean LDH level seen with UCB at $500 \mu \mathrm{g}$.

\section{Phagocytosis}

The phagocytic function of macrophages was measured by their uptake of $2 \mu \mathrm{m}$ fluorescent latex beads (fig 5). None of the particle types, instilled at $125 \mu \mathrm{g}$ had any significant effect on the ability of macrophages to phagocytose the indicator beads. However, at the higher dose of $500 \mu \mathrm{g}$ all particles were shown to significantly impair the phagocytic ability of the macrophage population $(\mathrm{p}<0.001)$.

\section{Chemotaxis}

The ability of macrophages to migrate towards the standard chemotaxin C5a (ZAS) is shown in fig 6. None of the particle types instilled at $125 \mu \mathrm{g}$ had any significant effect on the chemotactic ability of alveolar macrophages. However, at the

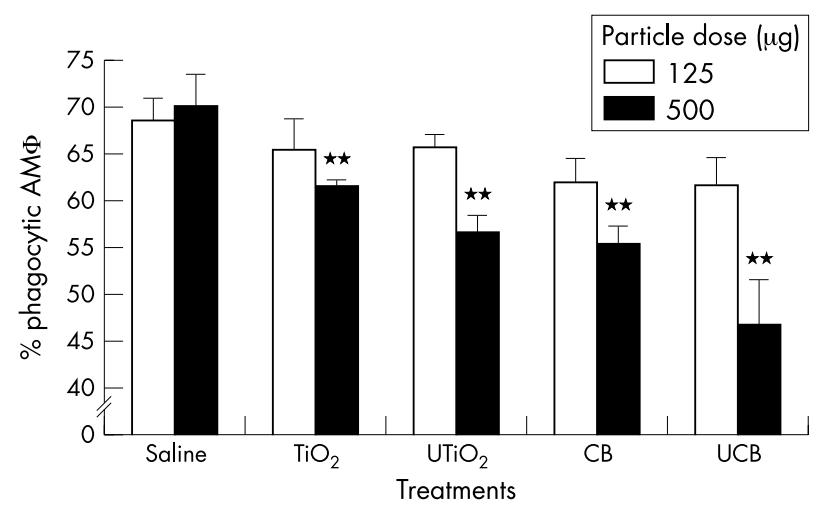

Figure 5 Percentage of phagocytic alveolar macrophages, 24 hours after instillation of test particles $(125 \mu \mathrm{g}$ or $500 \mu \mathrm{g})$. Results are the mean (SEM) of three separate experiments. Asterisks indicate significant differences from the saline control $\left({ }^{* *} p<0.001\right)$.

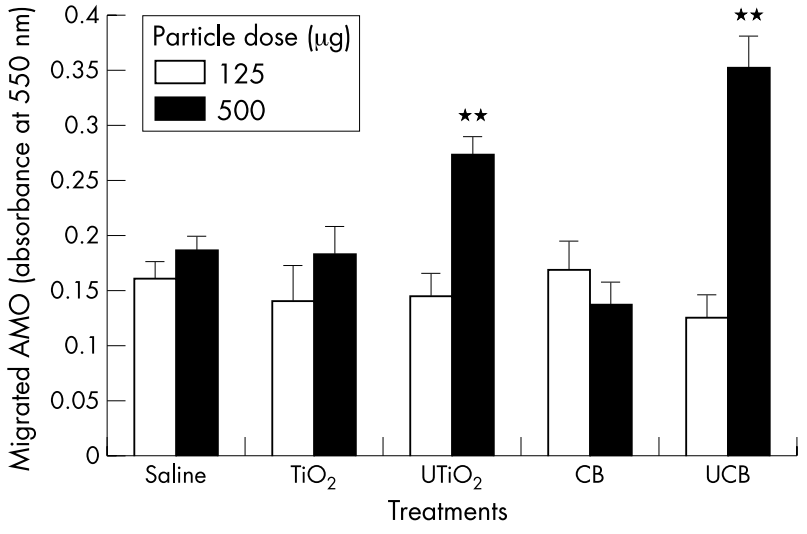

Figure 6 Chemotactic activity of alveolar macrophages, 24 hours after instillation of test particles $(125 \mu \mathrm{g}$ or $500 \mu \mathrm{g})$. Results are the mean (SEM) of three separate experiments. Asterisks indicate significant differences from the saline control $\left({ }^{* *} \mathrm{p}<0.001\right)$.

higher dose of $500 \mu \mathrm{g}$, treatment with both $\mathrm{UTiO}_{2}$ and UCB caused a significant increase in macrophage chemotaxis $(p<0.001)$, when compared to the saline control or the other test particles. In contrast, the instillation of $500 \mu \mathrm{g}$ of fine $\mathrm{TiO}_{2}$ and $\mathrm{CB}$ had no effect on macrophage mobility.

\section{DISCUSSION}

We showed that ultrafine or nanoparticles caused more inflammation than the same mass of fine particles, when instilled into rat lungs, and this was associated with more cell death, as shown by GGT, LDH, and protein levels in the BAL. All of the particle treatments caused decreased macrophage ability to phagocytose polystyrene beads. Macrophages from lungs of rats treated with ultrafine, but not fine particles, had increased ability to chemotact towards ZAS. Table 3 shows a summary of the data obtained in all of the assays and shows the remarkably greater effects of the ultrafines and highlights the greater activity of UCB compared to $\mathrm{UTiO}_{2}$.

This study supports the findings of several previous studies showing that ultrafine particles are highly inflammogenic. ${ }^{5}{ }^{14}$ This has been linked to their small size and large surface area with associated free radical generating systems. ${ }^{7-9}$ Transition metals have been implicated in the inflammogenicity of many iron containing dust types, including PMI0. ${ }^{15} 16$ However, the increased inflammatory response to UCB compared with $\mathrm{CB}$ could not be attributed to soluble transition metals, but was related to surface area or particle number. ${ }^{14}$ Further studies with different surface area doses of a range of low toxicity particle types has shown that the extent of inflammation is related to surface area dose in this instillation model. ${ }^{17}$ Particle number and surface area are closely related, but it is impossible to accurately describe particle number in these ultrafine samples in view of the tendency of ultrafine particles to aggregate into chains and clumps. Although we have only one measurement of surface area we also have data from the manufacturers regarding the surface area of CB and UCB, and this shows good agreement with our own figure.

In the present study, both ultrafines caused greater inflammation than the fine samples, but generally UCB induced a greater inflammatory response than $\mathrm{UTiO}_{2}$, as we have reported previously. ${ }^{18}$ UCB was also the only particulate to increase neutrophil recruitment at the lower dose. One explanation for this is that UCB has a much larger surface area than $\mathrm{UTiO}_{2}$ (table 1). This argues for the greater inflammatory effects seen with the ultrafines being attributable to a greater surface area. 
Table 3 Summary of the results obtained in the study

\begin{tabular}{|c|c|c|c|c|c|c|c|c|c|c|c|c|}
\hline & \multicolumn{2}{|c|}{ Inflammation } & \multicolumn{2}{|c|}{ LDH } & \multicolumn{2}{|c|}{ GGT } & \multicolumn{2}{|c|}{ Protein } & \multicolumn{2}{|c|}{ Phagocytosis } & \multicolumn{2}{|c|}{ Chemotaxis } \\
\hline & Lo & $\mathrm{Hi}$ & Lo & $\mathrm{Hi}$ & Lo & $\mathrm{Hi}$ & Lo & $\mathrm{Hi}$ & Lo & $\mathrm{Hi}$ & Lo & $\mathrm{Hi}$ \\
\hline$C B$ & NS & NS & NS & $\ln c$ & NS & NS & NS & NS & NS & Dec & NS & NS \\
\hline UCB & Inc & Inc & Inc & Inc & Inc & Inc & NS & Inc & NS & Dec & NS & Inc \\
\hline $\mathrm{TiO}_{2}$ & NS & NS & NS & NS & NS & NS & NS & NS & NS & Dec & NS & NS \\
\hline $\mathrm{UTiO}_{2}$ & NS & Inc & NS & Inc & NS & Inc & NS & Inc & NS & Dec & NS & Inc \\
\hline
\end{tabular}

Inc and Dec refer to significant changes compared to the control. NS, no significant difference from control.

Lo, $125 \mu \mathrm{g}$ dose; $\mathrm{Hi}, 500 \mu \mathrm{g}$ dose.

Epithelial injury appeared to be important in the proinflammatory effects of the ultrafines and there was close concordance between ability to cause inflammation, extent of epithelial injury as indicated by levels of GGT, total protein, and LDH in the lavage. Protein in lavage may emanate from the blood as transudation or exudation if there is inflammation, but may also result from increased local secretion in the airspaces. $\mathrm{C}$ reactive protein in BAL may be a better indicator of increased permeability. Previous studies with ultrafine $\mathrm{TiO}_{2}$ have suggested that the particles readily cross the epithelium, ${ }^{19}$ and in tracheal explants in organ culture, ultrafine $\mathrm{TiO}_{2}$ crossed the epithelium and entered the interstitium..$^{20}$ The data here suggest that there is preferential injury caused to the epithelium and this may assist the interstitial transfer of ultrafine particles.

The ultrafine particle types used here, $\mathrm{UTiO}_{2}$ and UCB, have been shown to induce oxidative stress and cytotoxicity in previous studies. ${ }^{9}{ }^{13}$ Brown and colleagues ${ }^{14}$ instilled rats with equal masses of ultrafine and fine $\mathrm{CB}$ and showed that UCB induced marked cytotoxicity and increased epithelial permeability, whereas CB had no or little effect. In addition, Afaq and colleagues ${ }^{21}$ reported that a single instillation of $\mathrm{UTiO}_{2}$ ( $2 \mathrm{mg}$ per rat) not only induced cytotoxicity, but there was also a progressive increase in hydrogen peroxide production and lipid peroxidation.

Additionally, the present study assessed the effect of ultrafine particles on two aspects of macrophage functionphagocytosis and chemotaxis. Macrophages are the cells that confront deposited particles and their ability to phagocytose, as well as their response to the phagocytic stimulus, determines the fate of the particles. ${ }^{22}$ For example, impaired ability to migrate could allow retention of particles in the lungs, with resultant build up of dose, while increased expression of pro-inflammatory cytokines could lead to inflammation..$^{22}$

We previously reported selective impairment of alveolar macrophage phagocytosis by ultrafines in vitro. ${ }^{11}$ We did not find any evidence that this occurred selectively in vivo at the doses used, since both fine and ultrafines at the highest dose caused impairment of phagocytosis. A study carried out by the authors in 1990 showed that pathogenic inflammogenic dusts could cause impairment of phagocytosis in vivo. ${ }^{23}$ Similar to our findings here, fine $\mathrm{TiO}_{2}$ did not have any effect, but coalmine dust, quartz, and asbestos did elicit macrophages in BAL with impaired ability to chemotact towards $\mathrm{C} 5 \mathrm{a},{ }^{23}$ the active chemoattractant generated when zymosan is used to treat serum and generate ZAS. ${ }^{24}$ However, in contrast to the present study, the 1990 one was an inhalation study. The present study suggests that ultrafines, in causing increased sensitivity to $\mathrm{C} 5 \mathrm{a}$, have a very different effect compared to any of these other pathogenic dusts. It should be noted, however, that the time point used here, one day, is very different to the long term inhalation exposure used in the 1990 study. ${ }^{23}$ It is possible that early response to harmful particles, seen here, is activation of macrophage chemotaxis to allow macrophages to migrate to sites of deposition with the evolved function of carrying out effective phagocytosis. In fact we have shown that macrophages are inhibited in their phagocytosis functions by ultrafines, ${ }^{25}$ and so in the case of ultrafines this key evolved function is subverted. In contrast to the increased responsiveness to chemotaxin seen here following acute exposure, chronic exposure, such as the 1990 study, culminates in impairment of chemotaxis.

The increased sensitivity of the macrophages from lungs inflamed by ultrafine particles to migrate towards a C5a chemotactic stimulus was a striking finding in the present study. This effect was absent in the fine particle treatments. Migration of the alveolar macrophage particle burden out of the lung is a crucial step in lung defence since it is the effective removal of "dose" of particles. Increased sensitivity to $\mathrm{C5}$ a could be seen as a response to the inflammatory milieu, but the low dose of $\mathrm{UTiO}_{2}$ also caused significant inflammation and the macrophages do not show any enhanced sensitivity to chemotaxin. Increased C5a sensitivity may then be seen as an effect additional to the inflammation and may be a result of the internal dose of ultrafine particles in the macrophages exposed to the higher dose.

Increased sensitivity to C5a could be seen as harmful in that some particle types activate complement at their sites of deposition in the lungs. ${ }^{26}$ Therefore any increased responsivity to this chemotaxin could retain macrophages in the lung, at these sites, hindering their clearance by the normal mucociliary route. Another source of $\mathrm{C} 5 \mathrm{a}$ could be the epithelial cells of the alveolar region; rat and human alveolar epithelial type II cells ${ }^{27}$ have been reported to constitutively express and secrete C5a. Further study into the cell biology of the increased sensitivity to $\mathrm{C} 5$ a of the macrophages exposed to the high dose of ultrafines is warranted.

In an additional experiment we investigated the chemotactic activity in the BAL fluids. There was no significant difference between the chemotactic activities of BAL fluids from particle treated animals and the saline control (data not shown). However, it is possible that this arose because of excessive dilution of chemotactic activity during BAL.

Ultrafine particles are a component of $\mathrm{PM}_{10}{ }^{28}$ and may play a role in its well documented toxicity. ${ }^{8}$ Numerous studies have shown that $\mathrm{PM}_{10}$ has oxidative activity ${ }^{29}$ that is capable of stimulating pro-inflammatory effects via oxidative stress that acts through a transition metal mediated mechanism. ${ }^{2}{ }^{30}$ We recently showed data to support the contention that ultrafine surfaces and transition metals can synergise in the production of oxidative stress and inflammation. The data described here provide another potential mechanism for the adverse effects of $\mathrm{PM}_{10}$, involving increased sensitivity to the chemotaxin $\mathrm{C} 5 \mathrm{a}$. In this regard some PM samples contain endotoxin $^{31}$ that can activate complement with the production of $\mathrm{C} 5 \mathrm{a}$ that could further contribute to the retention of macrophages, plus their particle burden, in the central area of the lungs, at sites of deposition. This accumulation of the $\mathrm{PM}_{10}$ dose in the lungs could be important in enhancing the response to $\mathrm{PM}_{10}$. 


\section{ACKNOWLEDGEMENTS}

LR was funded by the British Occupational Health Research Foundation.

\section{Authors' affiliations}

L C Renwick, D Brown, A Clouter, School of Life Sciences, Napier University, 10 Colinton Road, Edinburgh EH10 5DT, UK

K Donaldson, ELEGI Colt Laboratory, University of Edinburgh, Medical School, Teviot Place, Edinburgh EH8 9AG, UK

\section{REFERENCE}

1 Donaldson K, Stone V, Clouter A, et al. Ultrafine particles. Occup Environ Med 2001;58:211-16.

2 Lighty JS, Veranth JM, Sarofim AF. Combustion aerosols: factors governing their size and composition and implications to human health. J Air Waste Manag Assoc 2000;50:1565-618.

3 Avakian MD, Dellinger B, Fiedler $\mathrm{H}$, et al. The origin, fate, and health effects of combustion by-products: a research framework. Environ Health Perspect 2002; 110:1155-62.

4 Pui DH, Chen DR. Nanometer particles: a new frontier for multidisciplinary research. Journal of Aerosol Science 1997;28:481-760.

5 Ferin JOGPDP. Pulmonary retention of ultrafine and fine particles in rats. Am J Respir Cell Mol Biol 1992;6:535-42.

6 Zhang Q, Kusaka Y, Sato K, et al. Differences in the extent of inflammation caused by intratracheal exposure to three ultrafine metals: role of free radicals. J Toxicol Environ Health 1998;53:423-38.

7 Dick CA, Brown DM, Donaldson K, et al. The role of free radicals in the toxic and inflammatory effects of four different ultrafine particle types. Inhal Toxicol 2003;15:39-52.

8 Donaldson K, Brown D, Clouter A, et al. The pulmonary toxicology of ultrafine particles. J Aerosol Med 2002;15:213-220.

9 Wilson MR, Lightbody JH, Donaldson K, et al. Interactions between ultrafine particles and transition metals in vivo and in vitro. Toxicol Appl Pharmacol 2002; 184:172-9

10 Brunekreef B, Holgate ST. Air pollution and health. Lancet 2002;360:1233-42.

11 Renwick LC, Donaldson K, Clouter A. Impairment of alveolar macrophage phagocytosis by ultrafine particles. Toxicol Appl Pharmacol 2001;172:119-27.

1 la Harper RA, Stirling KM, Kreyling WG, et al. Intracellular particle dissolution in macrophages isolated from the lung of the Fisher (F-344) rat. Exp Lung Res 1994:20:143-56.

12 Clouter A, Houghton CE, Bowskill CA, et al. An in vitro/in vivo study into the short term effects of exposure to mineral fibres. Exp Toxicol Pathol 1996;48:484-6.

13 Brown DM, Wilson MR, MacNee W, et al. Size-dependent proinflammatory effects of ultrafine polystyrene particles: a role for surface area and oxidative stress in the enhanced activity of ultrafines. Toxicol Appl Pharmacol 2001;175:191-9.
14 Brown DM, Stone $V$, Findlay $P$, et al Increased inflammation and intracellular calcium caused by ultrafine carbon black is independent of transition metals or other soluble components [in process citation]. Occup Environ Med 2000;57:685-91.

15 Fubini B, Mollo L, Giamello E. Free-radical generation at the solid/liquid interface in iron-containing minerals. Free Radical Research 1995;23:593-614

16 Costa DL, Dreher KL. Bioavailable transition metals in particulate matter mediate cardiopulmonary injury in healthy and compromised animal models. Environ Health Perspect 1997; 105(suppl 5):1053-60.

17 Duffin R, Clouter A, Brown DM, et al. The importance of surface area and specific reactivity in the acute pulmonary inflammatory response to particles. Ann Occup Hyg 2002;46(suppl 1):242-5.

18 Dick CA, Brown DM Donaldson K, et al. The role of free radicals in the toxic and inflammatory effects of four different ultrafine particle types. Inhal Toxicol 2003; 15:39-52

19 Ferin J, Oberdorster G, Penney DP. Pulmonary retention of ultrafine and fine particles in rats. Am J Respir Cell Mol Biol 1992;6:535-42.

20 Churg A Stevens B, Wright JL. Comparison of the uptake of fine and ultrafine TiO2 in a tracheal explant system. Am J Physiol 1998;274:L81-6.

21 Afaq F, Abidi P, Matin R, et al. Cytotoxicity, pro-oxidant effects and antioxidant depletion in rat lung alveolar macrophages exposed to ultrafine titanium dioxide. J Appl Toxicol 1998;18:307-12.

22 Donaldson K, Tran CL. Inflammation caused by particles and fibres. Inhal Toxicol 2002; 14:5-27

23 Donaldson K, Brown GM, Brown DM, et al. Impaired chemotactic responses of bronchoalveolar leukocytes in experimental pneumoconiosis. J Pathol 1990;160:63-9.

24 Hatherill JR, Stephens KE, Nagao K, et al. Effects of anti-C5a antibodies on human polymorphonuclear leukocyte function: chemotaxis, chemiluminescence, and lysosomal enzyme release. J Biol Response Mod 1989;8:614-24.

25 Renwick LC, Donaldson K, Clouter A. Impairment of alveolar macrophage phagocytosis by ultrafine particles. Toxicol Appl Pharmacol 2001;172:119-27.

26 Governa M, Amati M, Valentino M, et al. In vitro cleavage by asbestos fibers of the fifth component of human complement through free-radical generation and kallikrein activation. J Toxicol Environ Health A 2000;59:539-52.

27 Strunk RC, Eidlen DM, Mason RJ. Pulmonary alveolar type II epithelial cells synthesize and secrete proteins of the classical and alternative complement pathways. J Clin Invest 1988:81:1419-26.

28 Ruuskanen J, Tuch T, Ten Brink $\mathrm{H}$, et al. Concentrations of ultrafine, fine and PM2.5 particles in three European cities. Atmospheric Environment 2001;35:3729-38.

29 Gilmour PS, Brown DM, Lindsay TG, et al. Adverse health effects of PM10 particles: involvement of iron in generation of hydroxyl radical. Occup Environ Med 1996;53:817-22

30 Jimenez LA, Thompson J, Brown DA, et al. Activation of NF-kappaB by $\mathrm{PM}(10)$ occurs via an iron-mediated mechanism in the absence of IkappaB degradation. Toxicol Appl Pharmacol 2000;166:101-10.

31 Becker S, Soukup JM, Gilmour MI, et al. Stimulation of human and rat alveolar macrophages by urban air particulates: effects on oxidant radical generation and cytokine production. Toxicol Appl Pharmacol 1996;141:637-48. 